\title{
Electrical pelvic floor stimulation: a possible alternative treatment for reflex urinary incontinence in patients with spinal cord injury
}

\author{
Manabu Ishigooka ${ }^{1}$, Tohru Hashimoto ${ }^{1}$, Shinsuke Hayami ${ }^{1}$, Yasuhiro Suzuki ${ }^{1}$, Teruhiro Nakada ${ }^{1}$ and Yasunobu \\ Handa $^{2}$ \\ ${ }^{1}$ Department of Urology, Yamagata University School of Medicine, Yamagata, Japan; ${ }^{2}$ Department of Restorative \\ neuromuscular Surgery and Rehabilitation, Tohoku University Graduate School of Medicine, Sendai, Japan
}

\begin{abstract}
The present study demonstrates the clinical experience of pelvic floor stimulation using percutaneous implantable electrodes and implantable electrical stimulator for the treatment of reflex urinary incontinence in patients with spinal cord injury. Pelvic floor stimulation was carried out on six paraplegic patients who had urinary incontinence from an overactive bladder. After the percutaneous implantation of a pair of electrodes, chronic stimulation was carried out by employing an implanted receiver or an external pulse regulator. Within 4 to 16 weeks of electrical stimulation urinary incontinence was improved in four of the six patients. In two of these six patients, incontinence was completely abolished subjectively. Urodynamic investigations demonstrated an increased volume at the first unstable contraction $(P<0.01)$ in all of the patients. Inhibition of detrusor overactivity was obtained from this procedure. The stimulation effect appeared to be constant during chronic stimulation. This new procedure probably provides a stable and reliable stimulation effect for long term treatment, and may be an alternative treatment for previous external electrical pelvic floor stimulation.
\end{abstract}

Keywords: urinary incontinence; spinal cord injury; electrical stimulation; pelvic floor musculature; perineal electrical stimulation

\section{Introduction}

It is now well known that electrical pelvic floor stimulation (EPFS) improves urinary incontinence due to an overactive bladder through the inhibitory spinal reflex mechanism. ${ }^{1-4}$ Previous reports demonstrated that the sympathetic nerve pathways would play a significant role in bladder inhibition by EPFS during urinary storage. ${ }^{4,5}$ Earlier EPFS has been performed by external stimulating devices, such as anal and/or vaginal plugs. ${ }^{6-9}$ Although EPFS by external devices offers a convenient treatment, there are several disadvantages. Leakage of electrical current from the device to the applied mucosa induces pain on stimulation, and occasionally injures the mucosa. Sufficient activation of the pelvic floor may depend upon the positioning of the device. Since sufficient contraction of pelvic floor muscles appears to be important for bladder inhibition, ${ }^{10}$ the effect of EPFS seems to be inadequate. To overcome such problems we previously described our preliminary experience of the percutaneous procedure for EPFS. ${ }^{11-13}$ In these studies, we employed a percutaneous implantable electrode, since this electrode appears to be suitable for chronic electrical stimulation. ${ }^{14,15}$ Moreover, in three patients in the present series, we performed chronic EPFS by a percutaneous implantable electrode

Correspondence: Manabu Ishigooka MD with an implantable stimulator which was regulated by an external pulse transmitter. The results of EPFS on patients with spinal cord injury are presented.

\section{Subjects and methods}

\section{Subjects}

Six male patients aged 29-51 years (average 36.8 years) underwent electrical pelvic floor stimulation. All of the patients had urinary incontinence resulting from spinal cord injury. Table 1 summarizes the clinical presentations of these cases. They all had full urological examinations including urodynamic studies. $\mathrm{H}_{2} \mathrm{O}$-cystometry revealed overactive detrusor function with detrusor external sphincter dyssynergia. Conservative treatment including self-catheterization and the administration of anticholinergic agents failed to control their incontinence.

\section{Electrode implantation}

The electrode we employed was a commercially available Teflon insulated 19 strand stainless steel wire (SES114, NEC, Japan). ${ }^{3,4}$ Before electrode implantation, test stimulation with a $25 \mathrm{G}$ thin needle electrode (SES115, NEC, Japan) was carried out. The technique for test stimulus and electrode implantation 
Table 1 Clinical presentations and results of EPFS in six patients with spinal cord injury

\begin{tabular}{|c|c|c|c|c|c|c|c|}
\hline Patient no. & Age & Sex & $\begin{array}{l}\text { Level of spinal } \\
\text { cord injury }\end{array}$ & $\begin{array}{c}\text { Method of bladder } \\
\text { drainage }\end{array}$ & $\begin{array}{l}\text { Implant of } \\
\text { stimulator }\end{array}$ & Effect of EPFS & $\begin{array}{l}\text { Treatment } \\
\text { period } \\
\text { (months) }\end{array}$ \\
\hline 1 & 34 & male & $\mathrm{Th} 12 / \mathrm{Ll}$ & $\mathrm{CIC}$ & No & Unchanged & 2 \\
\hline 2 & 51 & male & $\mathrm{C} 5 / 6$ & $\mathrm{CIC}$ & No & Improved & 28 \\
\hline 3 & 29 & male & $\mathrm{L} 1 / 2$ & CIC & No & Unchanged & 2 \\
\hline 4 & 24 & male & Th4/5 & CIC & Yes & Cured & 22 \\
\hline 5 & 40 & male & Th6/7 & Tapping & Yes & Improved & 16 \\
\hline 6 & 43 & male & $\mathrm{C} 4 / 5$ & Tapping & Yes & Unchanged & 2 \\
\hline
\end{tabular}
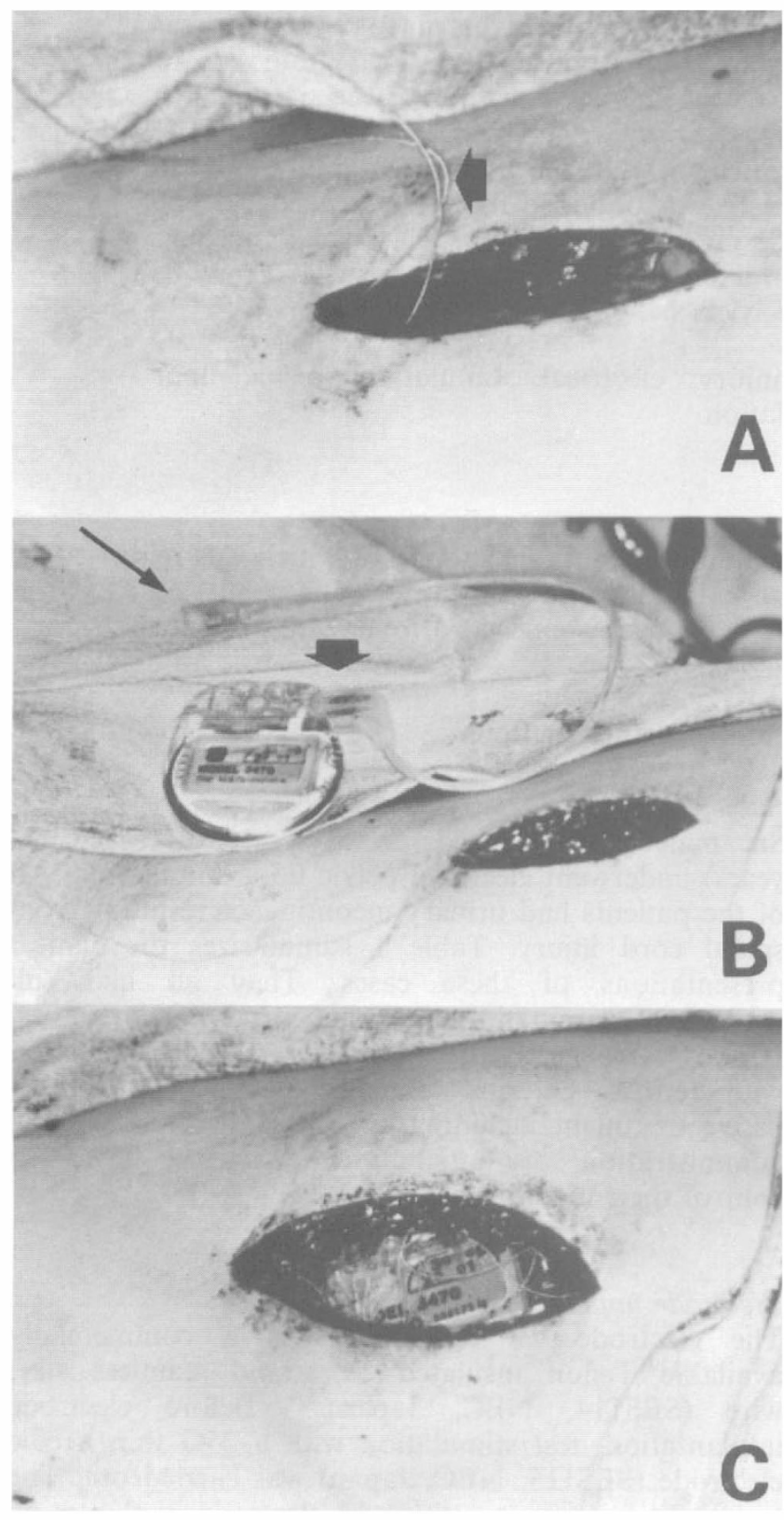

has been previously described in detail. ${ }^{11,12}$ Each patient had implanted stimulating electrodes in the perineal region, into the levator muscle or the external urethral sphincter. These two electrodes were buried in the subcutaneous tissue of the ipsilateral thigh using a subcutaneous 'tunnel' needle. ${ }^{12}$

\section{Implantation of the stimulating device}

In this series, three patients underwent implantation of the stimulator. To implant this, a longitudinal skin incision of about $6 \mathrm{~cm}$ was made in the medial aspect of the ipsilateral thigh. Electrodes were exposed through this skin incision (Figure 1). We used an implantable receiver (XTREL model 3470 with model 7496 extension, Medtronic, USA; Figure 2) which was developed as spinal cord stimulation for the control of neuralgia. ${ }^{16}$ Electrodes were attached to the stimulating device by a silicon adhesive agent (Figure 1). After opening the subcutaneous space by the blunt dissection, the stimulator was implanted, (Figure 1) and the subcutaneous tissue and skin were then closed. Implantation was accomplished within $40 \mathrm{~min}$ in all of the patients.

\section{Electrical stimulation}

Initiation of therapeutic stimulation took place 2 weeks after implantation to avoid the migration of electrodes. The stimulating wave form was a negative going rectangular pulse with a $0.5 \mathrm{msec}$ duration. We employed a fixed frequency of $5 \mathrm{~Hz}$ considering the bladder inhibition. ${ }^{17}$ The stimulating output was amplitude-modulated and its maximum voltage was

Figure 1 Procedure for the implantation. (A) Showing electrodes (arrow) exposed through the skin incision. (B) Electrodes were attached to the extension (thin arrow) and then connected to the receiver (arrow). (C) Electrodes, the extension and the receiver were implanted subcutaneously 
$10 \mathrm{~V}$. Patients received intermittent stimulation four times during daytime. Each stimulation lasting $30 \mathrm{~min}$. At night-time continuous stimulation was performed. Stimulation was regulated by an external radiofrequent pulse transmitter (XTREL model 3425, Medtronics, USA; Figure 2) in three patients during each stimulation period. The three other patients used our external pulse regulator for chronic stimulation, as we have previously reported. ${ }^{13}$ These patients required to connect the end of electrodes to the external stimulator during the stimulating periods.

\section{Evaluations}

Evaluations by water-cystometry were repeated every second week. In this series, the effect of EPFS was evaluated after 4 weeks of therapeutic stimulation. Bladder compliance was defined as a change in bladder volume per change in detrusor pressure.

Compliance was measured at the volume of first involuntary contraction. Changes in urodynamic parameters were analysed by Student's $t$-test. The degree of urinary incontinence was evaluated by the quantification of urine leakage by weighing urinary pad. Changes in subjective symptom were considered in three categories: cured, by which the patient stated he had no incontinence; improved, by which frequency of incontinence and/or quantity of urine leakage had been diminished but incontinence was still persistent; unchanged, by which subjective symptoms were not changed.

\section{Results}

Changes in urinary leakage

The results of chronic EPFS in six patients are shown in Table 1 and Figure 3A. In three of the six patients, 4 weeks of EPFS therapy improved their urinary incontinence. In two of these three patients, urinary incontinence has disappeared and they could manage

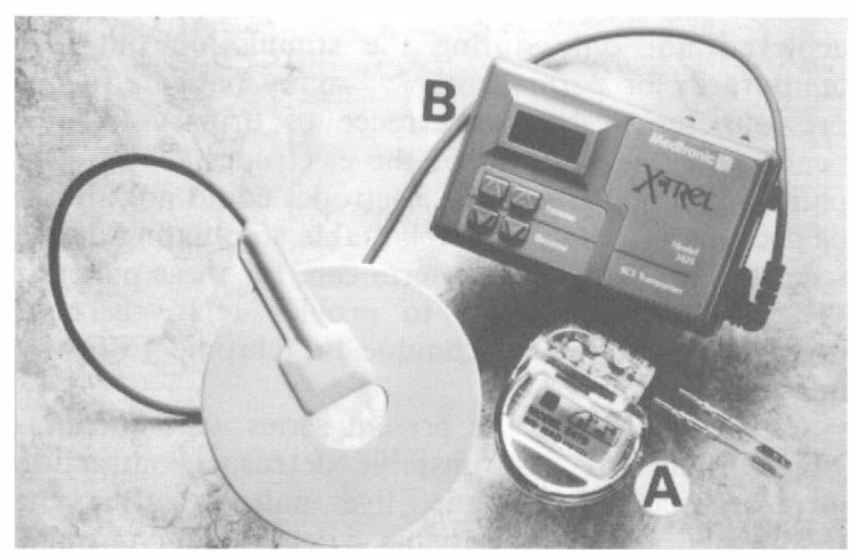

Figure 2 Showing implantable stimulator (receiver; A) and external pulse transmitter (B) without a urinary pad. In one patient, although he still requires a urinary pad, the amount of urinary leakage decreased. In three patients, urinary incontinence was not quantitatively improved by EPFS. Voiding by suprapubic tapping was not affected by EPFS. In this series, urinary incontinence was effectively treated in $50 \%$ of the patients.

\section{Urodynamic findings}

Figures $3 \mathrm{~B}-\mathrm{D}$ summarize the changes in the urodynamic parameters before, and after 4 weeks of treatment. The volume at first unstable contraction increased in five patients. The volumes at the first unstable contraction (mean $\pm \mathrm{SE}$ ) before and after the treatment were $129.8 \pm 25.7 \mathrm{ml}$ and $232.4 \pm 47.3 \mathrm{ml}$, respectively. This volume has also increased in two patients whose symptoms were unchanged. Detrusor pressure at the maximal unstable contraction had been remarkably suppressed in three out of six patients. The degree of unstable contraction was not significantly changed in the other three patients. Bladder compliance at the first unstable contraction appeared to be improved after the treatment. Figure 4 showed a representative response to EPFS.

\section{Adverse reactions and follow-up}

There were no complications related to the implantation of the stimulating device; and there were no problems concerned with the implantable stimulator.

Pain and discomfort on stimulation were resolved by lowering the stimulation amplitude. All patients could continue the stimulation during the period of
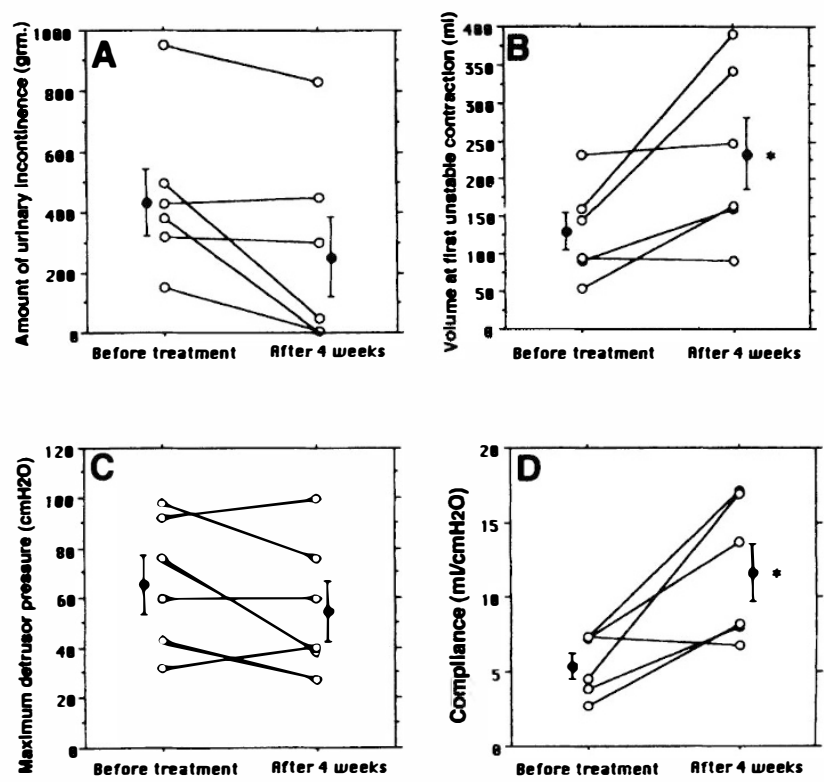

Figure 3 Changes in quantity of urine loss (A) and cystometric parameters (mean $\pm \mathrm{SE} ; \mathrm{B}-\mathrm{D}$ ) before and after therapeutic stimulation 
A

\section{Before treatment}
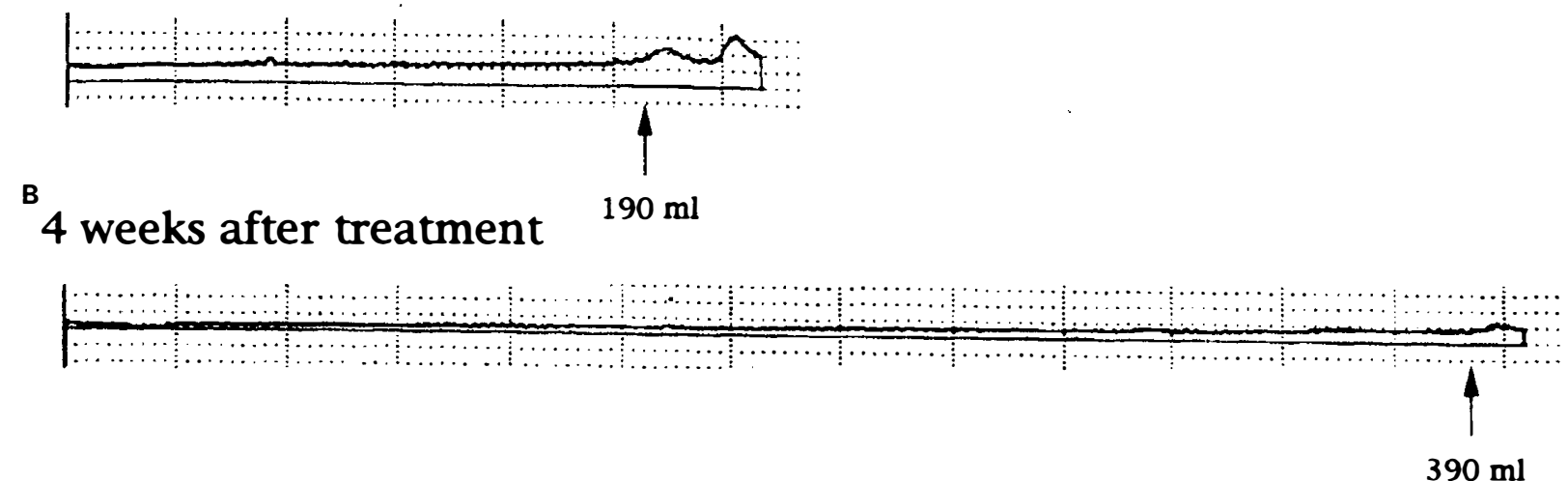

Figure 4 Changes in the findings by cystometry. (A) Before treatment. (B) Four weeks after the treatment. The volume of involuntary detrusor contraction increased from $190 \mathrm{ml}$ to $390 \mathrm{ml}$

treatment. The possible occurrence of electrode breakage or migration was checked by taking occasional Xrays. No electrode migrated. Stimulation is continued in three patients who respond well to the stimulation and discontinued in the other three patients at 8 weeks of treatment. The follow-up period of successfully treated patients are shown in Table 1. There were no apparent differences between stimulation by the implantable stimulator and the external stimulator in respect of the effects of treatment.

\section{Discussion}

In patients with spinal cord injury, voiding appears to be incomplete since they usually show detrusor-external sphincter dyssynergia. ${ }^{18}$ Moreover, detrusor hyperreflexia and high intravesical pressure are often associated with such a condition. Therefore, patients ordinarily show reflex urinary incontinence. The goal of bladder management in the patients with spinal cord injury is to achieve adequate bladder drainage, lowpressure urine storage, and low intravesical pressure. Relative continence and low-pressure urine storage can be made by administration of anti-cholinergic agent and intermittent catheterization. ${ }^{18}$ However, in many patients, urinary incontinence due to reflex bladder could not be managed by such treatments and they need to wear external collecting devices. Therefore, bladder inhibition by electrical stimulation appears to be applicable to achieve low-pressure urine storage and urinary continence in such patients.

Percutaneous implantable electrode had been developed for the use of functional electrical stimulation to restore impaired function of paralyzed extremities. ${ }^{14,15}$ Its fundamental properties and nonsurgical procedure appeared to be also eligible for the chronic application in EPFS. ${ }^{12,13}$ Indeed, as reported previously, our method could offer easy, stable and non-invasive treatment. ${ }^{12}$ In this series, we also implanted stimulating devices controlled by external radiofrequent pulse transmitter. Implantable electrode and stimulator for EPFS were also reported in the early 1960 s by Caldwell. ${ }^{19}$ However, this procedure was abandoned because of risk of major surgery for electrode implantation and limited durability of the device. Other investigators then tried external electrode arrangement. ${ }^{69}$ We could not abandon technique of direct stimulation, since direct procedure appears to offer more effective treatment than that of external stimulating system. ${ }^{20,21}$ Moreover, the effect of bladder suppression depends upon contraction of the external sphincter. ${ }^{10,22}$ EPFS by external electrode would not always produce adequate sphincteric contraction. Thus, we employed percutaneous direct implantation of electrodes. Although our method probably provides stable and sufficient stimulation by relatively non-invasive procedure, this method also has the following disadvantages: (1) Since we used external stimulator, patients must connect the electrodes to the external stimulator on each stimulation; (2) Since electrodes are exposed at the inside of the thigh, a fear for inflammation and cosmetic problems are inevitable; (3) Although, in general, bladder inhibition is achieved not only during the stimulation but also temporarily or permanently, ${ }^{4,23}$ many patients in our previous series show recurrence of urinary incontinence after the removal of the electrodes. ${ }^{13}$ For such patients, reimplantation of electrodes could not always be acceptable. Thus, an implantable stimulating device seemed necessary for long-term care for these patients. Present procedure seems to provide relatively noninvasive and durable technique for chronic EPFS by implantable devices.

Clinical results of the present series were encouraging. Volume at first unstable detrusor contraction increased considerably in five patients after the treatment. In two patients, degree of unstable contraction decreased. Clinical improvement rate of $50 \%$ in neuropathic overactive bladder seemed to be comparable to other reports. ${ }^{7,20,23}$ Low-pressure 
urinary storage and low-pressure bladder were achieved by EPFS. Serious complications by implantable stimulating devices did not appear in long term usage, although urinary incontinence in these patients could be managed by external collecting devices or urinary pad, such a management often results in dermatitis and skin ulceration of the perineal region. Improved urinary continence in these patients probably offers improved quality of life in such patients.

\section{Conclusions}

Urinary incontinence was improved in $50 \%$ of patients with spinal cord injury by EPFS. EPFS appears to be a physiological treatment, since this type of treatment utilizes normal spinal reflex mechanism. ${ }^{10,17}$ Furthermore, EPFS does not sacrifice other physiological functions. Although EPFS is not effective in all patients, this type of treatment shoud be considered on patients with reflex urinary incontinence if conservative therapies could not control their incontinence.

\section{References}

1 Sundin T, Carlsson C-A, Kock NG. Detrusor inhibition induced from mechanical stimulation of the anal region and from electrical stimulation of pudendal nerve afferents: An experimental study in cats. Invest Urol 1974; 11: 374 - 378.

2 Teague CT, Merrill DC. Electrical pelvic floor stimulation: Mechanism of action. Invest Urol 1977; 15: 65-69.

3 Fall M, Erlandson B-F, Nilson AE, Sundin T. Long-term intravaginal electrical stimulation in urge and stress incontinence. Scand J Urol Nephrol Suppl 1978; 44: 55 - 63

4 Lindstrom S, Fall M, Carlsson C-A, Erlandson BE. The neurophysiological basis of bladder inhibition in response to intravaginal electrical stimulation. J Urol 1983; 129: 405-410.

5 Ishigooka M, Hashimoto T, Sasagawa I, Nakada T. Reduction in norepinephrine content of the rabbit urinary bladder by alpha- 2 adrenergic antagonist after electrical pelvic floor stimulation. $J$ Urol 1994; 151: $774-775$.
6 Godec C, Cass AS, Ayala GF. Electrical stimulation for incontinence: Technique, selection and results. Urology 1976; 7 : $388-397$.

7 Fall M. Electrical pelvic floor stimulation for the control of detrusor instability. Neurourol Urodyn 1985; 4: 329-335.

8 Eriksen $\mathrm{BC}$, Mjonerod $\mathrm{OK}$. Changes in urodynamic measurement after succesful anal electrostimulation in female urinary incontinence. $\mathrm{Br} J$ Urol 1987; 59: 45-49.

9 Fossberg E et al. Maximal electrical stimulation in the treatment of unstable detrusor and urge incontinence. Eur Urol 1990; 18: 120123.

10 Tanagho EA. Concept of neuromodulation. Neurourol Urodyn $1993 ; 12: 487488$

11 Ishigooka $\mathrm{M}$ et al. Electrical pelvic floor stimulation in the management of urinary incontinence due to neuropathic overactive bladder. Frontiers Med Biol Engng 1993; 5: 1 -..-10.

12 Ishigooka $\mathrm{M}$ et al. A technique of percutaneous implantation for electrical pelvic floor stimulation. Eur Urol 1993; 23: 413-416.

13 Ishigooka $\mathrm{M}$ et al. Electrical pelvic floor stimulation by percutaneous implantable electrode. Br J Urol 1994; 74: 191 194.

14 Handa Y, Hoshimiya N. Functional electrical stimulation for the control of the upper extremities. Med Prog Technol 1987; 12: 51 63.

15 Handa Y, Hoshimiya N, Iguchi Y, Oda T. Development of percutaneous intramuscular electrode for multichannel FES system. IEEE Trans Biomed Eng 1989; 36: 705-710.

16 Spiegelman R, Friedman W. Spinal cord stimulation: A contemporary series. J Neurosurge 1991; 28: 65-71.

17 Fall M, Lindstrom S. Electrical stimulation: A physiological approach to the treatment of urinary incontinence. Urol Clin North Ame 1991; 18: 393-407.

18 Perkash I. Long-term urologic management of the patients with spinal cord injury. Urol Clin North Ame 1993; 20: $423-434$.

19 Caldwell KPS. Electrical control of sphincter incompetence. Lancet 1963; 2: $174-175$.

20 McGuir EJ, Shi-Chun Z, Horwinski ER, Lytton B. Treatment of motor and sensory detrusor instability by electrical stimulation. $J$ Urol 1983; 129: $78-79$

21 Ohlsson BL, Fall M, Franken-Sommar S. Effects of external and direct pudenal nerve maximal electrical stimulations in the treatment of the uninhibited overactive bladder. $\mathrm{Br} \mathrm{J}$ Urol 1989; 64: $374-380$.

22 Schmidt RA. Treatment of unstable bladder. Urology 1991; 37: $28-37$.

23 Plevnik S, Janez J. Maximal electrical stimulation for urinary incontinence: Report of 98 cases. Urology 1979; 14: 638-644. 